BRX TH-626

CALT 68-2811

\title{
The Bel-Robinson tensor for topologically massive gravity
}

\author{
S. Deser \\ Physics Department, Brandeis University, Waltham, MA 02454 and \\ Lauritsen Laboratory, California Institute of Technology, Pasadena, CA 91125 \\ deser@brandeis . edu \\ J. Franklin \\ Reed College, Portland, OR 97202 \\ jfrankli@reed.edu
}

\begin{abstract}
We construct, and establish the (covariant) conservation of, a 4-index "super-stress tensor" for topologically massive gravity (TMG). Separately, we discuss its invalidity in quadratic curvature models and suggest a generalization.
\end{abstract}

The 4-index Bel-Robinson tensor $B_{\gamma \mu \nu \rho}$, quadratic in the Riemann tensor and (covariantly) conserved on Einstein shell, has received much scrutiny in its original $D=4$ habitat (see references in [1]). There, $\mathrm{B}$ is the nearest thing to a covariant gravitational stress-tensor, for example playing essentially that role in permitting construction of higher $(L>2)$ loop local counter-terms in supergravity $[2,3]$. It also generalizes to $D>4$, at the minor price of losing tracelessness, like its spin 1 model, the Maxwell stress-tensor.

In this note, we turn to lower $D$, asking whether $\mathrm{B}$ survives in $D=3$ and if so, to what question is it the answer-in what theory, if any, is it conserved? Since the hallmark of $D=3$ is the identity of Riemann and Einstein tensors (they are double-duals), it is obvious that B vanishes identically on pure Einstein (i.e., flat space) shell], and becomes the trivial (and removable) constant tensor $\sim\left(\Lambda^{2} g_{\gamma \mu} g_{\nu \rho}+\right.$ symm $)$ in cosmological GR [4]. This leaves the dynamical hallmark of $D=3$, TMG [5], and the new quadratic curvature models [6,7], as the other possible beneficiaries. Our main result is that B both survives dimensional reduction and is conserved on TMG shell, in accord with the similar mechanism ensuring the Maxwell tensor's conservation on topologically massive electrodynamics (TME) shell. Separately, a simple argument shows why it does not work for generic quadratic curvature actions.

One obtains $\mathrm{B}$ in $D=3$ by inserting the Riemann-Ricci identites (we use de-densitized $\epsilon^{\mu \nu \alpha}$ throughout)

$$
R^{\mu \alpha \nu \beta} \equiv\left(g^{\mu \nu} R^{\alpha \beta}+\mathrm{symm}\right) \equiv \epsilon^{\mu \alpha \sigma} G_{\sigma \rho} \epsilon^{\nu \beta \rho}
$$

\footnotetext{
${ }^{1}$ Actually, B can already be made trivial on $D=4$ GR shell, by adding suitable terms [8].
} 
into a $D=4 \mathrm{~B}$. The resulting combination is:

$$
B_{\gamma \mu \nu \rho}=\bar{R}_{\mu \nu} \bar{R}_{\gamma \rho}+\bar{R}_{\mu \rho} \bar{R}_{\gamma \nu}-g_{\mu \gamma} \bar{R}_{\nu \beta} \bar{R}_{\rho}^{\beta}, \quad \bar{R}_{\mu \nu} \equiv R_{\mu \nu}-1 / 4 g_{\mu \nu} R
$$

the Schouten tensor $\bar{R}$ also defines the Cotton tensor below. B is manifestly symmetric under $(\gamma \mu, \nu \rho)$ pair interchanges (but not totally symmetric here because that depended on special $D=4$ identities). Clearly, B vanishes identically for $\bar{R}_{\mu \nu}=0$, and reduces to a constant tensor for the cosmological $\bar{R}_{\mu \nu}=\Lambda g_{\mu \nu}$ extension, a term which may even be removed by suitably adding to the definition of B there. Turning to TMG, its field equation is [5]

$$
G^{\mu \nu}=\mu^{-1} C^{\mu \nu} \equiv \mu^{-1} \epsilon^{\mu \rho \gamma} D_{\rho} \bar{R}_{\gamma}^{\nu}
$$

The Cotton tensor $C^{\mu \nu}$ is identically (covariantly) conserved, symmetric and traceless, so tracing (2) implies $R=0$, which simplifies on-shell calculations; $\mu$ is a constant with dimension of mass. [Our results will also apply to cosmologically extended TMG [9], much as they do for cosmological GR.] Our question then is whether B of (1) is conserved by virtue of (2). The reason we expect this is the close analogy between TMG and its vector version, TME. The latter model's abelian version (its non-abelian extension is similar), has (flat space) field equations resembling (2),

$$
\partial_{\beta} F^{\alpha \beta}=\frac{1}{2} \mu \epsilon^{\alpha \gamma \beta} F_{\gamma \beta} \equiv \mu^{*} F^{\alpha},
$$

while the analog of $\mathrm{B}$ is the Maxwell stress tensor

$$
T_{\mathrm{M} \mu \nu}=F_{\mu}{ }^{\beta} F_{\nu \beta}-1 / 4 g_{\mu \nu} F_{\alpha \beta} F^{\alpha \beta} .
$$

It is indeed conserved on TME shell, as follows:

$$
\partial_{\nu} T^{\mu \nu}=F^{\mu \beta} \partial_{\nu} F_{\beta}^{\nu}=\mu F^{\mu \beta *} F_{\beta} \equiv \mu \epsilon^{\mu \alpha \beta *} F_{\alpha}^{*} F_{\beta} \equiv 0 .
$$

This success motivates seeking a TMG chain similar to (5), schematically,

$$
D B \equiv R(D R-D R) \equiv R \epsilon C=\mu^{-1} \epsilon C C \stackrel{?}{\equiv} 0
$$

that is, we are hoping to set up a curl so as to use the algebraic identity $D_{\alpha} \bar{R}_{\beta \gamma}-D_{\gamma} \bar{R}_{\beta \alpha} \equiv \epsilon_{\mu \alpha \gamma} C_{\beta}^{\mu}$ as indicated. [There is a major distinction between the two models, however. The Maxwell tensor is also the stress tensor of TME since its Chern-Simons term, being metric-independent, does not contribute. Hence conservation is guaranteed a priori here [5], unlike the very existence, let alone conservation, of a B for TMG.] Taking the divergence of (1) and using (2) indeed yields

$$
D_{\gamma} B^{\gamma \mu \nu \rho}=\left[D^{\gamma} \bar{R}^{\mu \nu}-D^{\mu} \bar{R}^{\nu \gamma}\right] \bar{R}_{\gamma}{ }^{\rho}+\left[D^{\gamma} \bar{R}^{\mu \rho}-D^{\mu} \bar{R}^{\rho \gamma}\right] \bar{R}_{\gamma}{ }^{\nu}=\mu \epsilon^{\sigma \gamma \mu}\left(C_{\sigma}{ }^{\nu} C_{\gamma}{ }^{\rho}+C_{\sigma}{ }^{\rho} C_{\gamma}{ }^{\nu}\right) \equiv 0
$$

where the identity follows by the symmetry under $(\sigma \gamma)$. This establishes the nontrivial role of B as a "covariant" conserved gravitational tensor for TMG. It may thus find uses here similar to those of the original B in classifying GR solutions. Whether it is relevant to the quantum extensions of these theories is unclear, since $D=3$ GR is finite [10] and TMG may be [11].

The other gravitational model of special interest in $D=3$ is the "new quadratic curvature" theory. Its $L=a R+b \bar{R}^{2}$, or even its pure $\bar{R}^{2}$ variant, does not conserve $\mathrm{B}$. The reason is obvious 
and applies as well to all quadratic curvature actions in $D=4$. The divergence of (any) B behaves as $R D R$, while the $R^{2}$ field equations read $D D R+R R=0$, hence they do not tell us anything about $D R$. So unless $R D R$ vanishes for algebraic reasons, and it does not, there is no hope already at linearized, $D D R$, level, quite apart from the $R R$ terms. A clear example is the $\bar{R}^{2}$ field equation itself,

$$
\square \bar{R}_{\mu \nu}+\left(g_{\mu \nu} \square-\frac{3}{8} D_{\mu} D_{\nu}\right) R+\left(2 \bar{R}_{\mu \alpha} \bar{R}_{\nu}^{\alpha}-g_{\mu \nu} \bar{R}^{\alpha \beta} \bar{R}_{\alpha \beta}\right)=0 .
$$

B-nonconservation also makes physical sense: one would expect the correct candidate (if any) to have the form $B^{\prime}=D R D R$ to reflect the extra derivatives in $R^{2}$ actions.

In summary, we have obtained a conserved Bel-Robinson tensor for $D=3$ TMG, despite TMG's third derivative order. It is, gratifyingly, the reduction of one originally defined for $D=4$ GR, and fits nicely with the Maxwell stress tensor's conservation in TME. We also noted the unsuitability of $\mathrm{B}$ as a conserved tensor in quadratic curvature models, suggesting instead that a modified $B^{\prime} \sim D R D R$ might succeed.

SD acknowledges support from NSF PHY 07-57190 and DOE DE-FG02-164 92ER40701 grants.

\section{References}

[1] S. Deser, in "Gravitation and Relativity in General" (ed F. Atrio-Barandela and J. Martins), Wold Publishing (1999), gr-qc/9901007.

[2] S. Deser, K.S. Stelle and J.H. Kay, Phys. Rev. Lett., 38527 (1977).

[3] N. Beisert et al, Phys. Lett. B, 694265 (2010), hep-th/1009.1643.

[4] S. Deser and R. Jackiw, Ann. Phys. 153405 (1984).

[5] S.Deser, R.Jackiw and S.Templeton, Ann. Phys. 140372 (1982); Phys. Rev. Lett., 48975 (1982).

[6] E. A. Bergshoeff, O. Hohm and P. K. Townsend, Phys. Rev. Lett. 102 201301(2009), hepth/0901.1766.

[7] S. Deser, Phys. Rev. Lett. 103101302 (2009), hep-th/0904.4473.

[8] R.K. Sachs, Z. Phys. 157, 462 (1960).

[9] S. Deser, in "Quantum Theory of Gravity" (ed S.M. Christensen), Hilger London (1984).

[10] E. Witten, Nucl. Phys. B., 31146 (1988); S.Deser, J.McCarthy and Z.Yang, Phys. Lett. B, 22261 (1989).

[11] S. Deser and Z. Yang, Class. Quant. Grav., 71603 (1990). 